\title{
Real-Time Facial Expression Recognition App Development on Smart Phones
}

\author{
Humaid Alshamsi, Veton Kupuska \\ Electrical And Computer Engineering Department, Florida Institute Of Technology, Melbourne Fl, \\ USA
}

\begin{abstract}
Facial expression recognition (FER) is a crucial technology and a challenging task for human computer interaction. Previous method has been using different feature and classification method for FER and use basic method for testing. In this paper, we used the best feature descriptor for FER by empirically evaluating two feature descriptors, namely Facial Landmarks, and Binary Robust Independent Elementary Features (BRIEF) descriptors. We examine each feature descriptor by considering one classification method such as Support Vector Machine (SVM) with three unique facial expression recognition datasets. In addition to test accuracies, we present confusion matrixes of FER. We also analyze the effect of using this feature and image resolutions on FER performance. Our study indicates that Facial Landmarks descriptor works the best for FER to run on smart phone. The experimental results demonstrate that the proposed facial expression recognition on mobile phone is successful and it gives up to $96.27 \%$ recognition accuracy.

Keywords: Machine Learning; Facial Expression Recognition; Facial Landmarks; Image Processing; Mobile Computing.
\end{abstract}

\section{INTRODUCTION}

Facial expressions recognition (FER) has a great potential for improving our life quality. For instance, FER system is useful for medical application, such as when a person in or after the surgeon system can analysis behavior. FER system could also be used to security check method at airport to find out whether a person has significant change on emotion states; or when a suspect in under crime investigation. A robotic teacher could offer better learning experience by having a better understanding of student feeling.

Designing a facial expression recognition system that run on smart phone is the most difficult and challenging due to the huge number of images need to be test and stored, light condition, sunglasses, masks, and head pose. Over the years, researcher have proposed various techniques for computers to recognize human facial expression. Previous works typically focus on proposing basic feature descriptors and classification methods for facial expression recognition. In contrast, in this paper, we aim to identify the best feature descriptors by performing an extensive comparison study.

Facial expression movements have several roles in the interaction and communication between human beings. Visible facial movements are used to enhance and influence the emotion from speech. Moreover, facial actions are activated for a short time when the emotion passes. Thus, detecting the facial expression is an intuitive way to recognize emotion.

These technologies have a wide range of applicability from auto unlock in smart phones and psychological usage in the past few years [12]. It is a very good example on the success of computer vision on embedded devices. Facial expression recognition in such is a similar idea that will have numerous uses in the future. Facial expressions are one of those things that hold great importance to human in communication as they tend to convey emotions, energies and expressions without the use of words in communication. Darwin stated to the biological roots of facial expressions and the important role it plays in the survival of the species including human beings. Ekman [8] defined 6 basic emotional facial expressions such as happiness, sadness, anger, fear, disgust, and surprise and states how these expressions are universal. The deepest emotions are embedded in the facial expressions of human beings. Ekman states that all the expressions are a blend of all these basic expressions which sometimes makes it difficult to understand. Facial expressions basically function with the activation/dilatation of one or more of the total forty-three facial muscles.

\section{RELATED WORKS}

Facial analysis gained a lot of popularity in the efforts and research in the past years. The applications of facial analysis include recognition, expression analysis, simulation and in the animation industry [1, 2]. Humans naturally are equipped in recognizing emotions via facial expressions. Somewhat, universally six basic expressions are identifiable and research purpose. Paul Ekman and his colleagues announced the Facial Action Coding System (FACS) covering all possible ones in fixed 
images [3]. This system has been used vastly by many researchers, especially those with a psychological background and focus of interest and many facial expressions are coded by the FACS system. The FACS system is based on human observations and the labelling process is manual. For many years, researchers designed systems to classify facial expressions in an automated way. A lot of definitions of emotion have been given in previous years.

Numerous approaches were invented using still images and videos for facial expression recognition systems. The first to introduce local paramedic motion representations are Black and Yacoob then proceeding the information to the required classifier [4]. The low dimensional manifold technique for representing, trailing, and identifying facial expressions was created by Chang [5]. They utilized the Lipschitz embed system alignment for facial features for the manifold construction. The Gabor wavelets were subjugated to track the changes in facial expressions that were further examined temporal area for the expression recognition by Valstar and colleagues initially [6]. Some rare expressions of the face were studied using the templates of variable intensity by Kumano and his associates stating the differences between several classification templates [7]. The usage of Adaboost classifier and Support Vector machines rarely gained benefit after this [8]. Later on, Torre's methods were known for graphical spectral techniques in this field [9]. Zeng's understanding was prominent where the separation of non-emotional and emotional facial expression was validated [10]. Sometimes nonemotional are just commonly used habits of people. Recent research is focus on emotion recognition in the wild that was evidenced by the series of international challenges of EmotiW (2013[13], 2014[14] and 2015[15]) In these challenges, various automatic emotion recognition systems have been built to recognizing emotions from still images and short videos in a certain degree that might be used in real world applications. However, none of all these methods can be used in portable devices such as mobile phones in real-time.

\section{FACIAL EXPRESSION RECOGNITION}

The first step towards the design is the preprocessing which includes detection and tracking the face movements by using Hausdorff Distance for face detection [20]. Then the face image goes on the second step of feature extraction using Facial Landmarks method. The core element of the design is the feature extraction and classification. This is where support vector machines (SVM) algorithm is utilized to compare the histogram values with the stored values in the training set. The database used is the Cohn-Kanade $(\mathrm{CK}+)$ database [19], a very big database. Cohn Kanade's database is one of the wellknown databases of video recordings of facial expressions, Karolinska Directed Emotional Faces (KDEF) Dataset [16], and Japanese Female Facial Expression (JAFFE) Dataset [17]. The top of the facial expressions in those recordings is fully described by the activation of the Action Units (AUs).

Feature Descriptors: Haar descriptor [27] is one of the most popular used for facial expression recognition [28], [29]. Gabor descriptor [30] is one of the most common feature descriptors that has been used for FER [31], [32], [33], [34], [35]. BRIEF descriptor is also used for facial expression recognition [36]. HOG descriptor has been examined for facial expression recognition [37], [38], [39].In this study, we evaluate the performance a relatively new feature descriptor called facial landmarks descriptor [20] in facial expression recognition. To the best of our knowledge, we are the first to consider facial landmarks descriptor in real time facial expression recognition development on mobile phone.

Classification Methods: SVM classifier [33], [34] is the most common classifier that has been applied to facial expression recognition [40], [33], [34], [41], [42]. Several works have reported that SVM with linear kernel produce similar test outcomes when compared to radial basis function kernel (RBF) [33], [43], [34]. K-Nearest Neightbors (K-NN) classifier has also been used recently for facial expression recognition [44]. The results of the application are very accurate and very few errors till date. The databases are used for the testing of the proposed system and application is built on an Android platform and can run on any Android platform

\section{A. Overview of the System}

Figure 1 shows a block diagram of real time facial expression recognition running on mobile phone. The two main stages in the system are: (i) feature extraction and (ii) feature classification. The feature extraction stage involves pre-processing stages such as acquiring a sequence of images ( 8 frames/second) using a video camera and detecting the facial region of the image and standardizing the properties for lighting the image, this application used Facial Landmarks feature extraction. The feature classification process involves support vector machines (SVM) algorithm; it is widely accepted as a powerful nonparametric pattern classification method in image processing. 


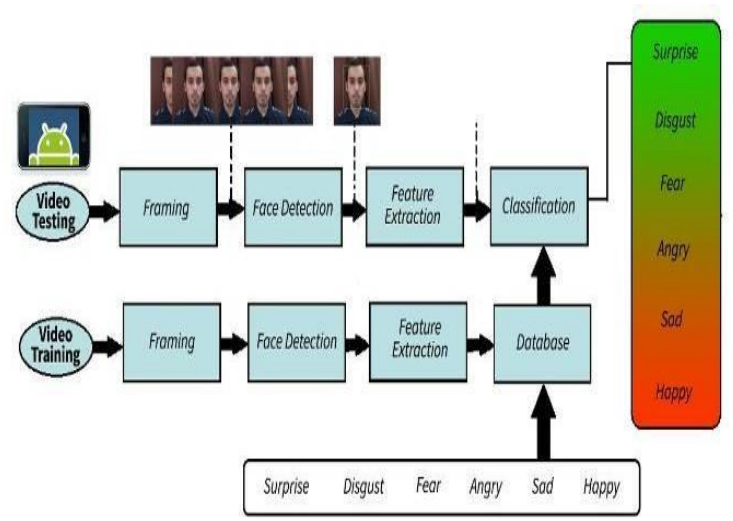

Figure 1. Overview structure of the facial expression

\section{B. Feature Extraction} recognition.

Facial landmarks are points on specific part of the facial image, which indicate for instance the location of the nose, the eyes, the brows and the mouth within an image. These points are tracked to follow the facial muscles' movements in time. If all facial landmarks are considered as a connected graph, we assume that the density of the graph differs in each facial expression (e.g. The pre-trained facial landmark detector inside the Dlib library is used to estimate the location of $68(\mathrm{x}, \mathrm{y})$-coordinates that map the facial structures on the face). After the detection of facial landmarks, distinctive information uses to extract from landmarks use "Center of Gravity (COG)" of all face landmarks is calculated (Formula $(1,2)$ ) and shown in figure $(2,3)$. Graphs are a very useful mathematical tool that can provide a wealth of information regarding the interrelationships of spatial points - in this case, of the facial landmarks. In order to extract features from these facial landmarks, spectral graph analysis is used, through which a characteristic vector, depicting areas of density in a graph, is extracted. To calculate the face offset correction by taking the tip of the nose and finding the angle for the nose as shown in figure (4) is calculated (Formal (3-8)).

$$
\begin{aligned}
& \mathrm{X}_{C O G}=\frac{1}{68} \sum_{i=1}^{68} x_{i} \\
& \mathrm{Y}_{C O G}=\frac{1}{68} \sum_{i=1}^{68} y_{i}
\end{aligned}
$$

Where $\mathrm{X}_{C O G}$ is $\mathrm{x}$-coordinate of $\mathrm{COG}$ and $\mathrm{Y}_{C O G}$ is $\mathrm{y}$ coordinate of COG.

$$
\begin{aligned}
& x_{\text {relative }_{i}}=x_{i}-\mathrm{X}_{C O G} \\
& y_{\text {relative }_{i}}=y_{i}-\mathrm{Y}_{C O G} \\
& E U C_{i}=\sqrt{\left(x_{i}-\mathrm{X}_{C O G}\right)^{2}+\left(y_{i}-Y_{C O G}\right)^{2}}(5) \\
& \beta_{\text {nose }}=\tan ^{-1}\left(\frac{y_{28}-y_{31}}{x_{28}-x_{31}}\right) \\
& \theta_{i}=\tan ^{-1}\left(\frac{y_{i}-y_{C O G}}{x_{i}-x_{C O G}}\right)-\beta_{\text {nose }}
\end{aligned}
$$

$$
F=\left\{x_{\text {relative }_{i}}, y_{\text {relative }_{i}}, E U C_{i}, \theta_{i}\right\} i=1,2, \ldots 8
$$

Where $E U C_{i}$ is Euclidean distance between point $i$ and central point, $\beta_{\text {nose }}$ is the angle of nose, point-28 top of the nose and point-31 tip of the nose. $\theta_{i}$ defines relative angle and is corrected when the face is not perfectly horizontal, and feature vector length is 272 .

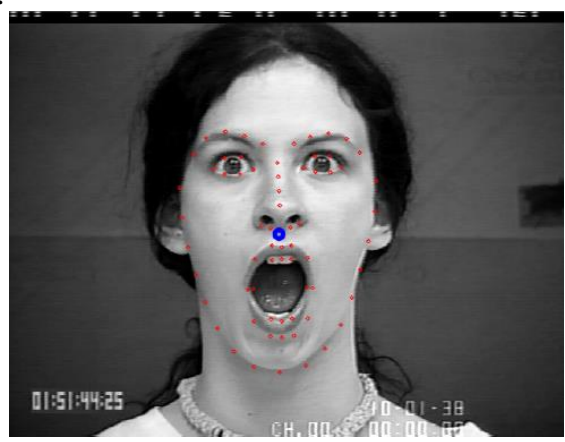

Figure 2. Facial landmarks and COG using $\mathrm{CK}+$ dataset.

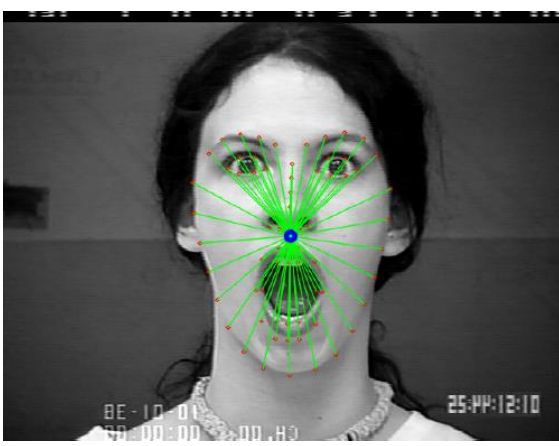

Figure 3. Line mapping between COG and Facial landmarks.

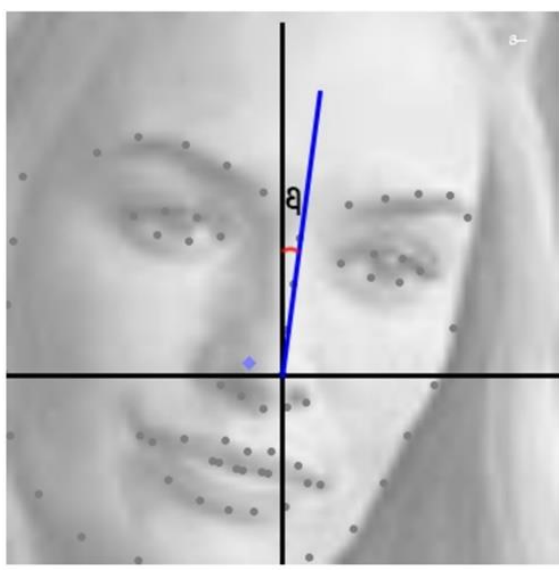

Figure 4. Face offset correction.

\section{Classification Method}

Support Vector Machine (SVM) has been widely used in various pattern recognition tasks. It is believed that SVM can achieve a near optimum separation among classes. In our study, we train SVMs to perform facial expression classification using the features we proposed. In general, SVM 
builds a hyperplane to separate the high dimensional space.

Support Vector Machines (SVMs) have been recently proposed by Vapnik and his co-workers [18] as a very effective method for general purpose pattern recognition. Intuitively, given a set of points belonging to two classes, an SVM finds the hyperplane that separates the largest possible fraction of points of the same class on the same side, while maximizing the distance from either class to the hyperplane. According to Vapnik [18], this hyperplane is called Optimal Separating Hyperplane (OSH) which minimizes the risk of misclassifying not only the examples in the training set but also the unseen examples of the test set.

Support vector machines (SVMs) [21] are originally designed for binary classification problem. How to effectively extend it for multi-class classification problem is still an on-going research issue. Several methods have been proposed where typically we construct a multi-class classifier by combining several binary classifiers. Some methods also have been proposed that consider all classes at once. As it is computationally more expensive to solve multi-class problems, comparisons of these methods using large-scale problems have not been seriously conducted yet.

The support vector machine [22], given labelled training data

$$
\begin{gathered}
D=\left\{\left(x_{i}, y_{i}\right)\right\}_{i=1}^{l}, x_{i} \in X \subset \mathbb{R}^{d}, y_{i} \in Y \\
=\{-1,+1\} \text { (9) }
\end{gathered}
$$

constructs a maximal margin linear classifier in a high dimensional feature space, $\Phi(x)$ defined by a positive definite kernel function, $\boldsymbol{k}\left(\boldsymbol{x}, \boldsymbol{x}^{\prime}\right)$, specifying an inner product in the feature space,

$$
\Phi(x) .\left(x^{\prime}\right)=k\left(x, x^{\prime}\right)
$$

A common kernel is the Gaussian radial basis function $(\mathrm{RBF})$

$$
k_{R B F}\left(x, x^{\prime}\right)=e^{-\gamma\left\|x-x^{\prime}\right\|^{2}}
$$

Where $\gamma>0$, kernel parameters to be decided by the users. Where Linear classifier is defined as

$$
h_{w, b}(x)=g\left(w^{T} x+b\right)
$$

Where $\boldsymbol{w}$ is weights and $\boldsymbol{b}$ is bias.

The discriminant function implemented by a support vector machine is given by

$$
f(x)=\left\{\sum_{i=1}^{l} \alpha_{i} y_{i} k\left(x_{i}, x\right)\right\}+b
$$

To find the optimal coefficients $\alpha$, of this expansion it is sufficient to maximize the functional,

$$
W(\alpha)=\sum_{i=1}^{l} \alpha_{i}-\frac{1}{2} \sum_{i, j=1}^{l} y_{i} y_{j} \alpha_{i} \alpha_{j} k\left(x_{i}, x_{j}\right)
$$

Which can be considered here as a cost function for optimization.
The popular SVM guide [26] suggests the following setting to train a kernel classifier.

1. Scale each feature to an interval like $[-1 ;+1]$.

2. Use Gaussian kernel. Choose $\gamma$; that gives the highest cross validation (CV) accuracy.

3. Obtain the model $\boldsymbol{w}$ using the selected $\gamma$.

\section{SYSTEM EVALUATION}

The proposed system was evaluated in a public dataset firstly. It will demonstrate its efficiency on facial expression and validate the method.

\section{Facial Expression Datasets}

Our experiment focuses on three facial expression datasets, namely Extended Cohn-Kanade $(\mathrm{CK}+)$ Facial Expression Dataset [23], [24], Japanese Female Facial Expression (JAFFE) dataset [17], and Karolinska Directed Emotional Faces (KDEF) dataset [16]. All faces in the three datasets were extracted with face detector of Computer Vision system in Android platform library.

\section{1) CK+ Dataset}

CK+ Database [23], [24] is one of the most widely used facial expression databases. It has facial expression images of 210 adults aged between 18-50 years old. Participants were consisted of $69 \%$ female. All participants were requested to perform a series of facial displays with the help from an instructor. With careful selection criterion, 327 sequences were identified as one of the seven discrete facial expression, namely (Happy, Sad, Angry, Fear, Surprise, Disgust, and Contempt). Each sequence begins with a neutral facial expression and end with a specific facial expression. For our research study, we excluded 'Contempt' expression and focused on the Basic-6 facial expression [25]. We select the first frame $f$ all sequences as 'Neutral' facial expression images and use the last frame from all sequences as basic-6 facial expression images. Overall, we use 636 facial expression images for our experiment study (Happy: 69, Sad: 28, Fear: 25, Disgust: 59, Neutral: 327, Surprise: 83, and Angry: 45). Figure 5, show some example images from CK+ Dataset, where red, orange, yellow, green, blue, indigo, and violet boxes represent angry, happy, fear, neutral, sad, surprise, and disgust facial expression recognition respectively.

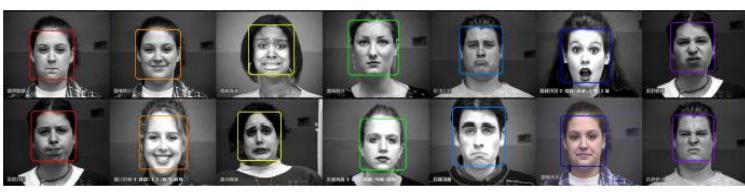

Figure 5. CK+ Facial Expression Dataset.

\section{2) JAFFE Dataset}

JAFFE Databases [17] contains 213 facial expression images of six basic facial expression and 
the neutral expression. All facial expression is taken from 10 Japanese female models. We use all 213 facial expression images for our experiment study (Happy: 31, Sad: 31, Fear: 32, Neutral: 30, Surprise: 30, and Angry: 30). Figure 6, show some example images from JAFFE Dataset, where red, orange, yellow, green, blue, indigo, and violet boxes represent angry, happy, fear, neutral, sad, surprise, and disgust facial expression recognition respectively.

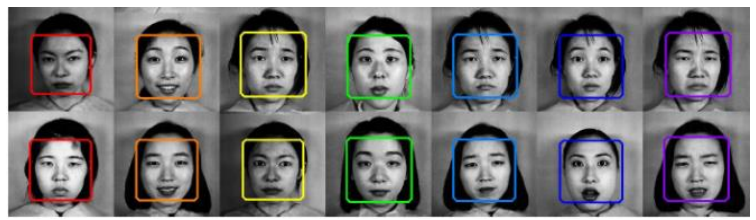

Figure 6. JAFFE Facial Expression Dataset.

\section{3) KDEF Dataset}

KDEF Database [16] records facial expression images from 140 amateur actors (70 males and 70 females) at 5 different viewing angles. All actors aged between 20-30 years old. They have no beards, no mustaches, no earrings, no eyeglasses, and mostly no visible make-up during phone sessions. For our testing study, we only consider frontal images, resulting in 980 facial expression images (Happy: 140, Sad: 140, Disgust:140, Angry:140, Neutral:140, Surprise: 140, Fear: 140). Figure 7, shows some example images from KDEF Dataset, where red, orange, yellow, green, blue, indigo, and violet boxes represent angry, happy, fear, neutral, sad, surprise, and disgust facial expression recognition respectively.

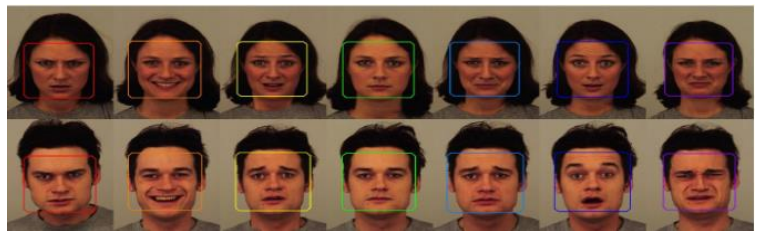

Figure 7. KDEF Facial Expression Dataset.

\section{E. Performance}

The performance of Facial Landmarks feature extracted and SVM algorithm has been examined. The result of this testing has been done within three different databases files. The following tables display the results that are collected from the three databases files. Table 1, demonstrates the system results of Cohn and Kanade $(\mathrm{CK}+)$ dataset which contains 636 images from 123 subjects, where 327 sequences are labeled with one of seven facial expressions, namely (angry, disgust, fear, happy, sad, surprise and contempt). Table 2, demonstrates the system results of Japanese Female Facial Expression (JAFFE) dataset which contains 213 images of 7 facial expressions posed by 10 Japanese female models. Table 3, demonstrates the system results of The
Karolinska Directed Emotional Faces (KDEF) dataset which contains 980 images of 7 facial expression.

Table 1. The Confusion Matrix Using Proposed Method on $\mathrm{CK}+$ Database. The overall recognition rate is $96.27 \%$.

\begin{tabular}{|l|c|c|c|c|c|c|c|}
\hline & N & F & S & H & S & A & D \\
\hline Natural (N) & 100 & 0 & 0 & 0 & 0 & 0 & 0 \\
\hline Fear(F) & 0 & 100 & 0 & 0 & 0 & 0 & 0 \\
\hline Surprise(S) & 0 & 0 & 100 & 0 & 0 & 0 & 0 \\
\hline Happy (H) & 0 & 0 & 0 & 100 & 0 & 0 & 0 \\
\hline Sad (S) & 0 & 0 & 0 & 0 & $\mathbf{8 7 . 5}$ & 0 & 12.5 \\
\hline Angry (A) & 0 & 0 & 0 & 0 & 0 & $\mathbf{9 2 . 3}$ & 7.6 \\
\hline Disgust (D) & 0 & 5.88 & 0 & 0 & 0 & 0 & 94.1 \\
\hline
\end{tabular}

TABLE 2. The Confusion Matrix Using Proposed Method on JAFFE Database. The overall recognition rate is $91.86 \%$.

\begin{tabular}{|l|c|c|c|c|c|c|c|}
\hline & N & F & S & H & S & A & D \\
\hline Natural(N) & $\mathbf{8 8 . 1}$ & 4.7 & 0 & 0 & 0 & 2.3 & 4.7 \\
\hline Fear(F) & 0 & $\mathbf{9 0 . 5}$ & 4.7 & 2.3 & 0 & 2.3 & 0 \\
\hline Surprise(S) & 4.7 & 0 & $\mathbf{9 0 . 4}$ & 4.7 & 0 & 0 & 0 \\
\hline Happy (H) & 2.4 & 0 & 0 & $\mathbf{9 2 . 8}$ & 2.3 & 2.3 & 0 \\
\hline Sad(S) & 0 & 4.7 & 2.3 & 2.3 & $\mathbf{9 0 . 4}$ & 0 & 0 \\
\hline Angry (A) & 2.3 & 2.3 & 0 & 2.3 & 0 & $\mathbf{9 2 . 8}$ & 0 \\
\hline Disgust (D) & 0 & 2.3 & 4.7 & 0 & 2.3 & 0 & $\mathbf{9 0 . 4}$ \\
\hline
\end{tabular}

TABLE 3. The Confusion Matrix Using Proposed Method on KDEF Database. The overall recognition rate is $90.82 \%$

\begin{tabular}{|l|c|c|c|c|c|c|c|} 
& N & F & S & H & S & A & D \\
\hline Natural (N) & 100 & 0 & 0 & 0 & 0 & 0 & 0 \\
\hline Fear(F) & 11.1 & 88.9 & 0 & 0 & 0 & 0 & 0 \\
\hline Surprise(S) & 11.1 & 0 & 88.9 & 0 & 0 & 0 & 0 \\
\hline Happy (H) & 0 & 0 & 0 & 88.9 & 11.1 & 0 & 0 \\
\hline Sad (S) & 0 & 0 & 0 & 0 & 100 & 0 & 0 \\
\hline Angry (A) & 0 & 11.11 & 0 & 0 & 0 & $\mathbf{8 8 . 9}$ & 0 \\
\hline Disgust (D) & 0 & 0 & 0 & 0 & 12.5 & 0 & $\mathbf{8 7 . 5}$ \\
\hline
\end{tabular}

\section{IMPLEMENTATION ON THE PHONE F. Feature Extraction}

This his section of the report pertains to the part of the code related to feature extraction and method which has been utilized to extract the features of the images. After extracting the features of the images, these features are stored to make a database so that it can further be utilized for testing purposes.

When the main body of the code is run, it asks for the training images (using $70 \%$ as training images) and then asks for the test images (using 30\% as testing images). When the images are loaded in the system, these images presented to feature extraction module. 


\section{G. Testing of the System}

The developed training database was tested on the test images to see whether the database is formed correctly and the system is working well. In order to test the system, there must be some classifier to compare the test images with that of training images database. Testing has been done by developing code in MATLAB and was tested on three different databases CK+ dataset, JAFFE dataset and KDEF dataset respectively. Following is the code which is used to implement the testing using SVM classifier and the results will be depicted.

\section{H. Testing on Matlab and the Phone}

Landmarks + COG descriptor has emerged as an important feature descriptor. Its value lies in its computational complexity as well as its recognition rate in most cases. A variant of the Landmarks + COG descriptor named Landmarks + COG -64 (i.e. 64 bytes descriptor) has been implemented. It has been shown in $[5,7]$ that a 64 bytes descriptor is sufficient to obtain very good matching results. The input for feature extraction is the region of interest i.e. the face. This region is converted to grayscale. Gaussian smoothing [5, 7] is applied on the resulting image i.e. it is filtered using a Gaussian low-pass filter with variance of Gaussian kernel taken as 2 [5, 7] and window size of $8 \times 8$ [5].The testing of Landmarks + COG descriptors works on pixel level and is thus very sensitive to noise. By pre-smoothing the patch, this sensitivity can be reduced, increasing the stability and repeatability of the descriptors. MATLAB simulation result of filtrating is shown in Figure $[9,10]$.

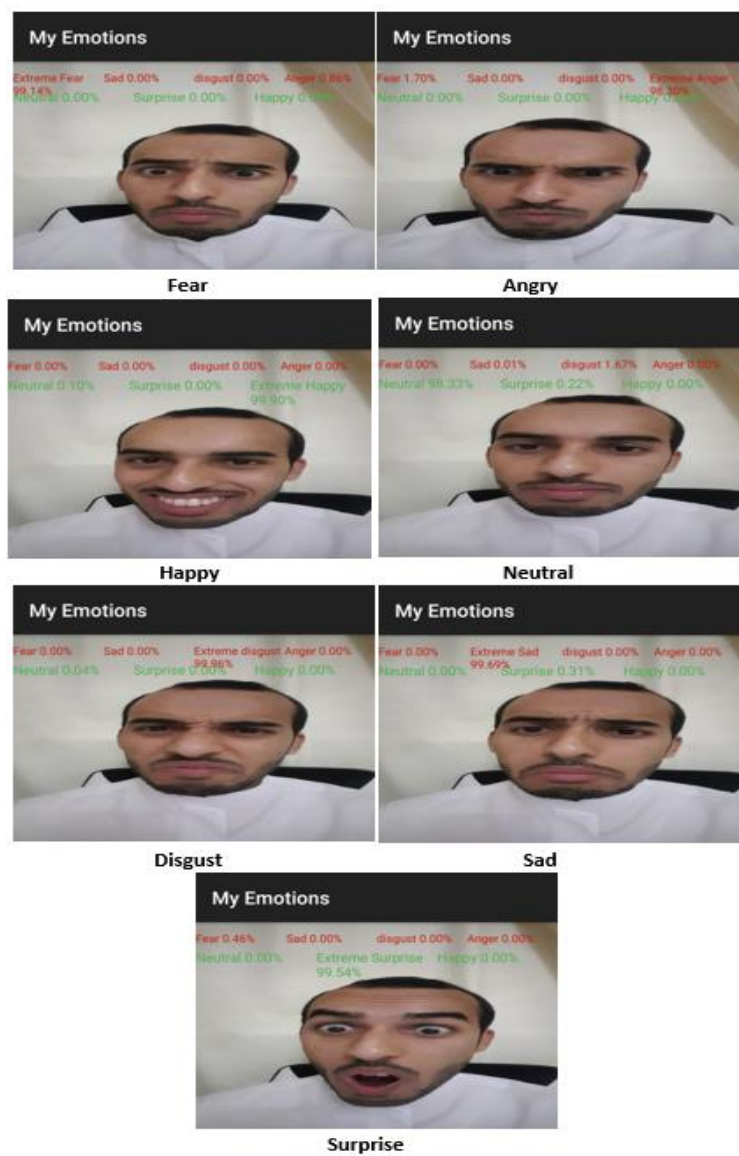

Figure 8. Testing result from the mobile phone.

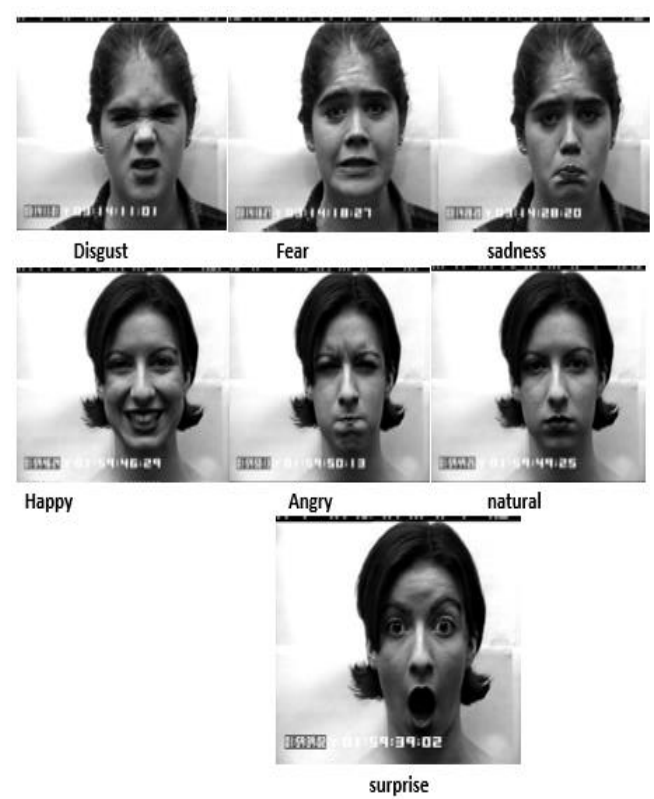

Figure 9. Example from $\mathrm{CK}+$ dataset after Extract the feature using Matlab. 


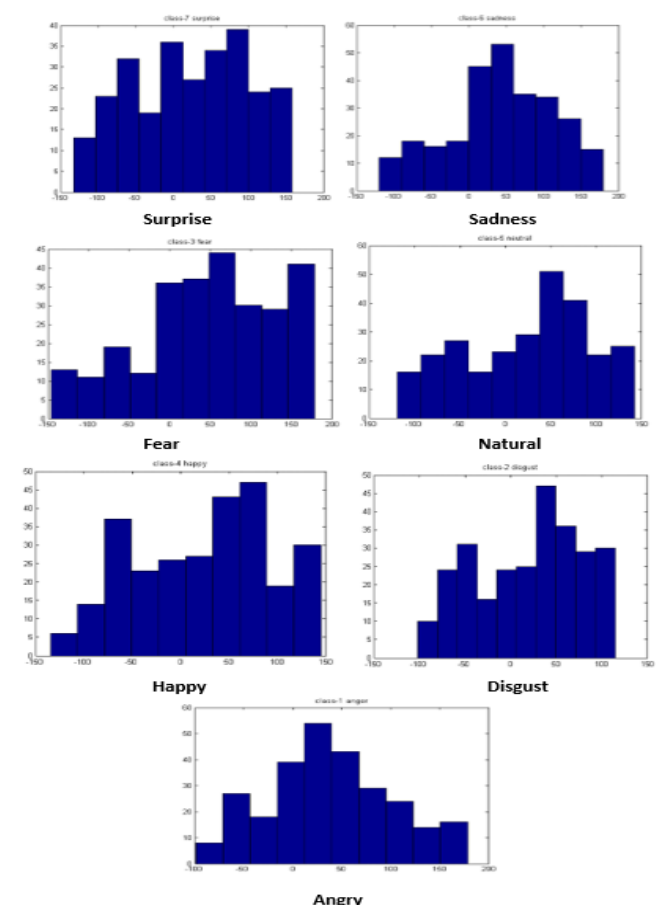

Figure 10. Histogram of Facial Landmarks descriptors using CK+ dataset show all seven FER.

Histogram of the values of Facial Landmarks descriptors for all the patches is then plotted to visualize the values of the features in a graphical manner. The number of bins can be varied as per requirement.

The absence of a blue color in any bin out of 10 means there is no element in that bin. A bar of height 1 means that one element of the specified column exists in that particular bin. Similarly, a bar of height 2 means that two elements of the specified column exist in that particular bin and so on. A sample histogram is shown in figure 10. Due to the randomness in selection of two points for binary tests, the resulting histogram is bound to show different values on different runs of the software but statistically they represent the same image.

\section{Testing summary}

The final step in this project is to assess and evaluate the project performance; to measure how many of the requirements for emotional face recognition on mobile phones can be achieved. Actually, testing has been continuously addressed from the early implementation stage until the final stage.

Firstly, the testing of each function is carried out individually. It is tested to ensure that the algorithm and each line code works correctly. Sometimes, a small sample of the image data is used to test the code and sometimes a different kind of data set is built. For example, a database of numeric matrix is built to calculate easily the results of the tested function manually and to compare the target results with the code results.

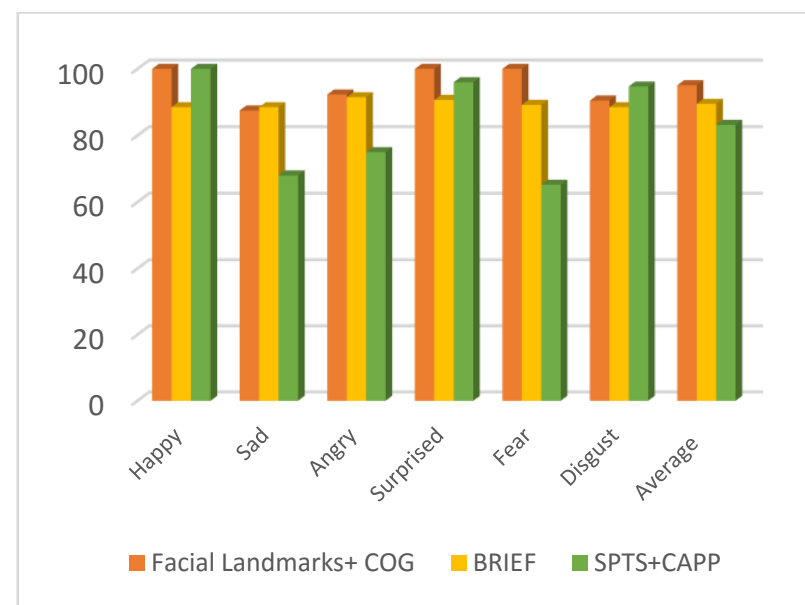

Figure 11. Performance comparison on Facial Landmarks+COG, BRIEF and SPTS+CAPP features.

Secondly, after completing a certain stage, the performance of that stage is tested. Furthermore, after integrating the system stages, the overall system performance was tested. In these phases, sometimes an implemented algorithm that is not useful for this project is discovered; then one is forced to return to the starting point. For example, the support vector machines (SVM) algorithm is described three times in three different papers. All these attempts failed in our hands. The problem was with the huge number of multi-class features that need to be trained. To solve this problem, attention was turned to the Android platform tools and a MATLAB tool that can be used with the project data. The Android platform was used to program the application and the MATLAB tool used to test the application. Eventually, after many attempts, the optimal solution was found.

TABLE 4. Compare Proposed Method with Other Two Methods.

\begin{tabular}{|l|c|c|c|}
\hline & Proposed method & BRIEF & SPTS+CAPP \\
\hline Happy & 100 & 88.5 & 100 \\
\hline Sad & 87.5 & 88.5 & 68 \\
\hline Angry & 92.3 & 91.5 & 75 \\
\hline Surprised & 100 & 90.7 & 96 \\
\hline Fear & 100 & 89.2 & 65.2 \\
\hline Disgust & 94.12 & 88.5 & 94.7 \\
\hline Natural & 100 & - & - \\
\hline Average & 96.3 & 89.5 & 83.2 \\
\hline
\end{tabular}

Finally, the entire project was tested to guarantee that the project met the requirements and achieved its aim. This is the most significant step, which ensures that the study algorithm is efficient and powerful; that it could recognize emotional face expressions from a mobile phone and it is much better than previous work that been test before by using BRIEF method [45]. 


\section{CONCLUSION AND DISCUSSION}

By comparing the results of these three methods, it is evident that my system developed using Facial Landmarks+ COG feature outperforms the other as its results were more that $85 \%$ in the detection range while in the other paper the average detection rate is about $66.7 \%$ for CAPP features and around $53.7 \%$ for SPTS features (results are for the first six emotions). When they combined the features (SPTS + CAPP) the detection rate of the system was increased to around $83.2 \%$ but it was still lower than our system [9]. By using hybrid features detection rates can be increased. While comparing each emotion detection results of system (using Facial Landmarks+ COG features) with these given in the reference (using BRIEF features and using SPTS + CAPP features) following results are obtained: Angry - 92.3\% (91.5\%, 75\%), Disgust - 90.5\% (88.5\%, $94.7 \%)$, Fear - 100\% (89.2\%, 65.2\%), Happy - 100\% $(8.5 \%, 100 \%)$, Sadness - 87.5\% (88.5\%, 68\%), Surprised $-100 \%(90.7 \%, 96 \%)$ respectively. As we see from individual emotion results that in most of the emotions the referenced system outperforms ours, but overall their average is a little lower than out result and the average for these six emotions is 95.1\%. Table 4; shows the comparison between the two work that been explained above.

Although the proposed method has been successfully implemented on smart phone for realtime facial expression recognition and a satisfied performance has been achieved, the work can be improved further by adding new features such as graphs that display the performance of the system while running on real-time and show the facial expression. It can also be very interesting to try run the system using could computing server. The classifier should be run real-time on the smart phone, but the training can be down offline.

\section{REFERENCES}

[1] Pantic, M., Pentland, A., Nijholt, A., Huang, T.S.: Human Computing and Machine Understanding of Human Behavior: A Survey, in Artificial Intelligence for Human Computing, 2007.

[2] Li, S.Z., Jain, A.K.: Handbook of Face Recognition, ISBN: 0-387-40595-X, 2005.

[3] Ekman, P.: Strong evidence for universals in facial expressions:a reply to Russell's mistaken critique. Psychol. Bull. 115(2), 268-287, 1994.

[4] Black, M. J., Yacoob, Y.: Recognizing facial expressions in image sequences using local parameterized models of image motion. Inter. Journal of CV, 25(1), pp. 23--48, 1997.

[5] Hu, C., Chang, Y., Feris, R., Turk. M.: Manifold based analysis of facial expression, Image and Vision Computing 24, pp. 60--614, 2006.

[6] Valstar, M.F., Pantic, M.: Fully automatic facial action unit detection and temporal analysis. Proceedings of IEEE Int'l Conf. Computer Vision and Pattern Recognition, 2006.
[7] Kumano, S., Otsuka, K., Yamato, J., Eisaku, S., Sata, Y.: Pose-Invariant facial expression recognition using variable intensity templates. Asian Conf. on Computer Vision, 2007.

[8] Bartlett, S., Littlewort, G., Frank, G., Lainscsek, C., Fasel, I., Movellan, J.: Fully automatic facial action recognition in spontaneous behavior. In Proc. Conf. Automatic Face \& Gesture Recognition, pp. 223--230, 2006.

[9] Torre, F., Campoy, J., Ambadar, Z., Cohn, J.F.: Temporal Segmentation of Facial Behavior, International Conference on Computer Vision, October, 2007.

[10] Zeng, Z., Fu, Y., Roisman, G.I., Zhen, W.: Spontaneous emotional facial expression detection. Journal of Multimedia, 2006.

[11] Sakarkaya, M.; Yanbol, F.; Kurt, Z., "Comparison of several classification algorithms for gender recognition from face images," Intelligent Engineering Systems (INES), 2012 IEEE 16th International Conference on , vol., no., pp.97,101, 13-15 June 2012.

[12] Dieter Findling, Rainhard, and Rene Mayrhofer. "Towards Pan Shot Face Unlock". Int J of Pervasive Comp \& Comm 9.3 (2013): 190-208. Web.

[13] Dhall, R. Goecke, J. Joshi, M. Wagner and T. Gedeon, Emotion Recognition In The Wild Challenge 2013, ACM ICMI 2013.

[14] A. Dhall, R. Goecke, J. Joshi, K. Sikka and T. Gedeon, Emotion Recognition In The Wild Challenge 2014: Baseline, Data and Protocol, ACM ICMI 2014.

[15] Dhall, Abhinav et al. "Video And Image Based Emotion Recognition Challenges In The Wild: Emotiw 2015". ResearchGate. N.p., 2015. Web.

[16] Lundqvist, D., Flykt, A. and Ohman, A.: The Karolinska Directed Emotional Faces - KDEF, CD ROM from Department of Clinical Neu-roscience, Psychology section, Karolinska Institutet.

[17] Lyons, M., Budynek, J. and Akamatsu, S.: Automatic Classification of Single Facial Images, IEEE Trans. Pattern Analysis and Machine Intelligence, Vol.21, No.12, pp.1357-1362 (1999).

[18] C. Cortes and V. Vapnik, "Support-vector network," In Machine Learning, 1995, pp. 273-297.

[19] "The Extended Cohn-Kanade Dataset (CK+): A Complete Dataset For Action Unit And EmotionSpecified Expression". N.p., 2016.

[20] Gheorghita Ghinea, Rajkumar Kannan, and Suresh kannaiyan,"Gradient-Orientation-Based PCA Subspace for Novel Face Recognition"in IEEE Access, vol 2,2169-3536, 2014.

[21] G. Dai and C. Zhou, "Face Recognition Using Support Vector Machines with the Robust Feature," In Proc. of IEEE workshop on Robot and Human Interactive Communication, 2003, pp. 49-53.

[22] P. J. Phillips, "Support Vector Machines Applied to Face Recognition," In Advances in Neural Information Processing Systems 11, MIT Press, 1999, pp. 803-809.

[23] Kanade, T., Cohn, J. and Tian, Y.: Comprehensive Database for Fa-cial Expression Analysis, Proc. 4th International Conference on Au-tomatic Face and Gesture Recognition, pp.46-53 (2000).

[24] Lucey, P., Cohn, J., Kanade, T., Saragih, J., Ambadar, Z. and Matthews, I.: The Extended Cohn-Kanade Dataset $(\mathrm{CK}+)$ : A Com-plete Dataset for Action Unit and Emotion-specified Expression, Proc. IEEE Computer Society Conference on Computer Vision and Pattern Recognition Workshops, pp.94-101 (2010). 
[25] Ekman, P.: An Argument for Basic Emotions, Cognition and Emotion, Vol.6, pp.169-200 (1992).

[26] C.-W. Hsu, C.-C. Chang, and C.-J. Lin. A practical guide to support vector classification. Technical report, National Taiwan University, 2003

[27] Papageorgiou, C., Oren, M. and Poggio, T.: A General Framework for Object Detection, Proc. 6th International Conference on Computer Vision, pp.555$562(1998)$

[28] Xu, C., Dong, C., Feng, Z. and Cao, T.: Facial Expression Pervasive Analysis Based on Haar-Like Features and SVM, Proc. The Interna-tional Conference on E-business Technology and Strategy, pp.521- 529 (2012).

[29] Jung, S., Kim, D., An, K. and Chung, M.: Efficient Rectangle Fea-ture Extraction for Real-time Facial Expression Recognition based on AdaBoost, Proc. IEEE/RSJ International Conference on Intelligent Robots and Systems, pp.1941-1946 (2005).

[30] Gabor, D.: Theory of Communication, Journal of the Institution of Electrical Engineers, Vol.93, No.26, pp.429-457 (1946).

[31] Zhang, Z., Lyons, M., Schuster, M. and Akamatsu, S.: Comparison between Geometry-based and Gaborwavelets-based Facial Expres-sion Recognition using Multi-layer Perceptron, Proc. 3rd IEEE In-ternational Conference on Automatic Face and Gesture Recognition, pp.454-459 (1998).

[32] Gonzalez, I., Sahli, H., Enescu, V. and Verhelst, W.: Context-independent Facial Action Unit Recognition Using Shape and Gabor Phase Information, Proc. 4th International Conference on Affective Computing and Intelligent Interaction, pp.548-557 (2011).

[33] Wu, T., Bartlett, M. and Movellan, J.: Facial Expression Recognition Using Gabor Motion Energy Filters, Proc. IEEE Computer Society Conference on Computer Vision and Pattern Recognition Workshops, pp.42-47 (2010).

[34] Bartlett, M., Littlewort, G., Lainscsek, C., Fasel, I., Frank, M. and Movellan, J.: Fully Automatic Facial Action Recognition in Spon-taneous Behavior, Proc. 7th International Conference on Automatic Face and Gesture Recognition, pp.223-228 (2006).

[35] Lee, C. and Shih, C.: Gabor Feature Selection for Facial Expression Recognition, Proc. International Conference on Signals and Elec-tronic Systems, pp.139-142 (2010).

[36] Ozuysal, M., Calonder, M., Lepetit, V. and Fua, P.: Fast Keypoint Recognition Using Random Ferns, IEEE Trans. Pattern Analysis and Machine Intelligence, Vol.32, No.3, pp.448-461 (2010).

[37] Dalal, N. and Triggs, B.: Histograms of Oriented Gradients for Human Detection, Proc. IEEE Computer Society Conference on Computer Vi-sion and Pattern Recognition, pp.886-893 (2005)

[38] Dahmane, M. and Meunier, J.: Emotion Recognition using Dynamic Grid-based HoG Features, Proc. IEEE International Conference on Automatic Face and Gesture Recognition Workshops, pp.884-888 (2011)

[39] Orrite, C., Ganan, A. and Rogez, G.: HOG-Based Decision Tree for Facial Expression Classification, Proc. 4th Iberian Conference on Pat-tern Recognition and Image Analysis, pp.176-183 (2009).

[40] Shan, C., Gong, S. and McOwan, P.: Facial Expression Recognition based on Local Binary Patterns: A Comprehensive Study, Journal of Image and Vision Computing, Vol.27, No.6, pp.803-816 (2009).

[41] Littlewort, G., Bartlett, M., Fasel, I., Susskind, J. and Movellan, J.: Dynamics of Facial Expression Extracted Automatically from Video, Journal of Image and Vision Computing, Vol.24, No.6, pp.615-625 (2006)

[42] Nagi, G., Rahmat, R., Khalid, F. and Taufik, M.: Region-Based Fa-cial Expression Recognition in Still Images, Journal of Information Processing Systems, Vol.9, No.1, pp.173-188 (2013).

[43] Bartlett, M., Littlewort, G., Frank, M., Lainscsek, C., Fasel, I. and Movellan, J.: Automatic Recognition of Facial Actions in Sponta-neous Expressions, Journal of Multimedia, Vol.1, No.6, pp.22-35 (2006).

[44] Eleftheriadis, S., Rudovic, O. and Pantic, M.: Discriminative Shared Gaussian Processes for Multiview and View-Invariant Facial Ex-pression Recognition, IEEE Trans. Image Processing, Vol.24, No.1, pp.189-204 (2015).

[45] H. Alshamsi, V. Këpuska, and H. Meng, "Real Time Facial Expression Recognition App Development on Mobile Phones," The International Journal of Science \& Technology, vol. 5, no. 10, pp. 507-512, Oct. 2016. 